\title{
Preparation and Evaluation of Pralidoxime-Loaded PLGA Nanoparticles as Potential Carriers of the Drug across the Blood Brain Barrier
}

\author{
Washington Chigumira, ${ }^{1}$ Prosper Maposa, ${ }^{1}$ Louis L. Gadaga, ${ }^{1}$ Admire Dube, ${ }^{2}$ \\ Dexter Tagwireyi, ${ }^{1}$ and Charles C. Maponga ${ }^{1}$ \\ ${ }^{1}$ Drug and Toxicology Information Service (DaTIS), School of Pharmacy, College of Health Sciences, University of Zimbabwe, \\ P.O. Box A 178, Avondale, Harare, Zimbabwe \\ ${ }^{2}$ School of Pharmacy, University of Western Cape, Robert Sobukwe Road, Bellville 7535, South Africa
}

Correspondence should be addressed to Prosper Maposa; promaposa@gmail.com

Received 15 May 2015; Accepted 24 June 2015

Academic Editor: Anh-Tuan Le

Copyright (C) 2015 Washington Chigumira et al. This is an open access article distributed under the Creative Commons Attribution License, which permits unrestricted use, distribution, and reproduction in any medium, provided the original work is properly cited.

Pralidoxime is an organophosphate antidote with poor central nervous system distribution due to a high polarity. In the present study, pralidoxime-loaded poly(lactic-co-glycolic acid) nanoparticles were prepared and evaluated as a potential delivery system of the drug into the central nervous system. The nanoparticles were prepared using double emulsion solvent evaporation method. Poly(lactic-co-glycolic acid) (PLGA) in ethyl acetate made the organic phase and pralidoxime in water made the aqueous phase. The system was stabilized by polyvinyl alcohol. Different drug/polymer ratios were used $(1: 1,1: 2$, and $1: 4)$ and the fabricated particles were characterized for encapsulation efficiency using UV-VIS Spectroscopy; particle size distribution, polydispersity index, and zeta potential using photon correlation spectroscopy; and in vitro drug release profile using UV-VIS Spectroscopy. Mean particle sizes were $386.6 \mathrm{~nm}, 304.7 \mathrm{~nm}$, and $322.8 \mathrm{~nm}$, encapsulation efficiency was $28.58 \%, 51.91 \%$, and $68.78 \%$, and zeta potential was $5.04 \mathrm{mV}$, $3.31 \mathrm{mV}$, and $5.98 \mathrm{mV}$ for particles with drug/polymer ratios $1: 1,1: 2$, and $1: 4$, respectively. In vitro drug release profile changed from biphasic to monobasic as the drug/polymer ratio decreased from 1:1 to 1:4. Stable pralidoxime-loaded PLGA nanoparticles were produced using double emulsion solvent evaporation techniques.

\section{Introduction}

Organophosphates (OP) intoxication accounts for the highest number of poisoning cases across the globe $[1,2]$. It is estimated that about three million people are exposed to organophosphates each year, with up to three hundred thousand fatalities $[1,2]$. Severe poisoning affects peripheral and central nervous systems and eventually causes paralysis of body extremities and respiratory muscle. The major cause of death in severe poisoning cases is respiratory failure due to depression of central nervous system (CNS) respiratory center, neuromuscular weakness (mainly the diaphragm muscle), and excessive bronchosecretions [3].

Despite the wide use of organophosphates [4] and high OP intoxication incidences worldwide [1], the management of severe acute OP poisoning cases is still a challenge with the available drugs. Pralidoxime was reported to be of great benefit in reversing respiratory symptoms of OP poisoning [5] but its use is limited due to a poor blood brain barrier penetration [6]. Organophosphates, on the contrary, can freely pass the barrier resulting in various CNS poisoning effects. Improved CNS distribution of oximes can potentially improve the drug effectiveness in the management of organophosphate poisoning through reversal of the nicotinic and muscarinic receptor effects both peripherally and centrally.

Efforts to overcome the blood brain barrier (BBB) have focused on altering either the barrier integrity and characteristics or the drug properties. Nanoparticles might be a better technique to circumvent the BBB since no $\mathrm{BBB}$ or 
drug manipulation is necessary. Poly(lactic-co-glycolic acid) PLGA nanoparticles have proved to improve the blood brain barrier penetration of a number of drugs that are poorly distributed in the CNS [7]. The current study is aimed at fabrication and analyses of pralidoxime-loaded PLGA nanoparticles, using different drug/polymer ratios, for potential use in the delivery of the drug into the central nervous system.

\section{Materials and Methods}

2.1. Materials. Pralidoxime chloride (pyridine-2-aldoxime methochloride) (RD grade), PLGA (50:50), ethyl acetate, and polyvinyl alcohol (PVA) were obtained from Sigma Aldrich Company, Germany. Disodium orthophosphate, citric acid, potassium dihydrogen orthophosphate, sodium hydroxide $(\mathrm{NaOH})$, and sodium chloride $(\mathrm{NaCl})$ were obtained from Associated Chemical Enterprise (South Africa). Distilled water was used in all the experiments. The following equipment was used during the study: $\mathrm{pH}$ meter (Jenway, UK), vortex mixer (Heidolph Reax 2000, Germany), magnetic stirring plate (Sigma Aldrich, Germany), sonicator (Westwood Ultrasonics, UK), ultracentrifuge (Beckman Optima LE-80k Ultracentrifuge, USA and Hermle Z160M, Germany), UV-VIS Spectrophotometer (Shimadzu, Japan), and Zetasizer (Malvern Zetasizer Nano ZS90, UK).

\subsection{Fabrication of Pralidoxime-Loaded PLGA Nanoparti-} cles. Pralidoxime-loaded PLGA nanoparticles were prepared using a double emulsion solvent evaporation method. $1.2 \mathrm{~mL}$ aqueous pralidoxime solution $(25 \mathrm{mg} / \mathrm{mL}, 50 \mathrm{mg} / \mathrm{mL}$, or $100 \mathrm{mg} / \mathrm{mL}$ ) was emulsified in $8 \mathrm{~mL}$ of ethyl acetate containing PLGA (120 mg) by means of homogenization on an ice bath at a speed of $13000 \mathrm{rpm}$ for 90 seconds. The primary emulsion was further emulsified in $32 \mathrm{~mL}$ of $2 \%$ (w/v) PVA solution containing $5 \%(\mathrm{w} / \mathrm{v})$ of $\mathrm{NaCl}$ by homogenization at $25000 \mathrm{rpm}$ for 10 minutes on an ice bath. The resultant double emulsion was stirred for 4 hours at $25^{\circ} \mathrm{C}$ on a magnetic stirring plate at $500 \mathrm{rpm}$. The nanoparticle suspension was then kept under refrigeration overnight. The nanoparticles were recovered by ultracentrifugation at $13,400 \mathrm{rpm}$ for 30 minutes at $4^{\circ} \mathrm{C}$. Following this, nanoparticle sediments were washed thrice with water then lyophilized overnight.

2.3. Encapsulation Efficiency. The nanoparticles were destroyed by acetonitrile in $1 \mathrm{~mL}$ eppendorf tubes under a vortex mixer, immediately after the washing step of fabrication (before lyophilization). The resultant solution was passed through a membrane filter $(0.22 \mu \mathrm{m}$ pore size $)$ and the amount of pralidoxime contained in the destroyed nanoparticles was determined by a UV-VIS spectrophotometer at $263 \mathrm{~nm}$. The amount of pralidoxime loaded in the nanoparticles was determined by measuring the amount of the drug encapsulated per $\mathrm{mL}$ of nanoparticle suspension,
TABLE 1: Encapsulation efficiency of pralidoxime-loaded PLGA nanoparticles with different drug/polymer ratios.

\begin{tabular}{lcc}
\hline $\begin{array}{l}\text { Drug/polymer } \\
\text { ratio }\end{array}$ & $\begin{array}{c}\text { Loading efficiency } \\
(\mathrm{mg} / \mathrm{mL})\end{array}$ & $\begin{array}{c}\text { Encapsulation efficiency } \\
(\%)\end{array}$ \\
\hline $1: 1$ & 0.98 & $28.58 \pm 0.01$ \\
$1: 2$ & 0.89 & $51.91 \pm 0.02$ \\
$1: 4$ & 0.59 & $68.78 \pm 0.03$ \\
\hline
\end{tabular}

Encapsulation efficiency is recorded as mean \pm SD.

which was done in triplicates. Encapsulation efficiency was calculated according to the following formula:

$$
\begin{aligned}
& \text { Encapsulation Efficiency }(\mathrm{EE}) \\
& \qquad=\frac{\text { Amt of pralidoximed entrapped }}{\text { total amt of pralidoxime added }} \times 100 \% .
\end{aligned}
$$

2.4. Particle Size, Polydispersity Index, and Zeta Potential. The particle size distribution, polydispersity index, and zeta potential of the pralidoxime-loaded PLGA nanoparticles were determined in triplicates by a photon correlation spectroscopy (PCS) using a zetasizer (Malvern Zetasizer Nano ZS90, UK). Approximately, $1 \mathrm{mg}$ of each sample was dissolved in $1 \mathrm{~mL}$ of deionized water. The dissolved sample was sonicated for 30 minutes. The samples were placed in a zetasizer and the particle size, polydispersity index, and zeta potential were then observed.

2.5. In Vitro Drug Release Analysis. The in vitro pralidoxime release from the PLGA nanoparticles was carried out at $37^{\circ} \mathrm{C}$ in simulated blood fluid ( $\mathrm{pH}$ 7.4). The buffer solution was prepared according to the United States Pharmacopoeia (USP 2007). $1 \mathrm{~mL}$ phosphate buffer solution ( $\mathrm{pH} 7.4)$ was placed in eppendorf tubes (containing the washed nanoparticle samples) sufficient for all the time points required. The eppendorf tubes were placed in a water bath at $37^{\circ} \mathrm{C}$. The samples were withdrawn for analysis after 1.0, 2.0, 3.0, 4.0, 6.0 , and 24.0 hours. The absorbance of the solutions was determined in triplicates by UV spectroscopy at $263 \mathrm{~nm}$.

2.6. Analysis of Results. ANOVA was done on the results using GraphPad Prism 5. All the statistical tests were done at $95 \%$ level of significance.

\section{Results}

3.1. Encapsulation Efficiency. Nanoparticles with drug/ polymer ratio of $1: 4$ had highest encapsulation efficiency $(68.78 \%)$ and the encapsulation efficiency decreased with increase in drug/polymer ratio as illustrated in Table 1. The differences in encapsulation efficiency among nanoparticles with different drug/polymer ratios were statistically significant at 5\% standard error $(P=0.0001$ for all the combinations).

3.2. Particles Size and Polydispersity Index (PDI). The results for the particle size, polydispersity, and zeta potential are 
TABLE 2: Mean particle size, polydispersity index (PDI), and zeta potential for pralidoxime-loaded PLGA nanoparticles with different drug : polymer ratio.

\begin{tabular}{lccc}
\hline $\begin{array}{l}\text { Drug/polymer } \\
\text { ratio }\end{array}$ & $\begin{array}{c}\text { Particle size } \\
(\mathrm{nm})\end{array}$ & $\begin{array}{c}\text { Zeta potential } \\
(\mathrm{mV})\end{array}$ & PDI \\
\hline $1: 1$ & $386.6 \pm 15.33$ & $5.04 \pm 0.35$ & $0.323 \pm 0.021$ \\
$1: 2$ & $304.7 \pm 7.167$ & $3.31 \pm 0.27$ & $0.180 \pm 0.032$ \\
$1: 4$ & $322.8 \pm 2.193$ & $5.98 \pm 0.30$ & $0.203 \pm 0.001$ \\
\hline
\end{tabular}

Data represented as mean $\pm \mathrm{SD}$.

shown in Table 2. The least mean particle size observed was $304.7 \mathrm{~nm}$ for the particles with drug/polymer ratio $1: 2$, followed by $322.8 \mathrm{~nm}$ for drug/polymer ratio 1:4 but the difference was statistically insignificant at $5 \%$ standard error $(P=0.3540)$. The particles with drug/polymer ratio $1: 1$ were the largest $(386.6 \mathrm{~nm})$ and statistically different from the others at 5\% standard error $(P=0.001$ for all the combinations).

Polydispersity index was less than 0.5 for all the three samples. The particles with drug/polymer ratios $1: 2$ and $1: 4$ had the least PDIs and their difference at 5\% standard error was insignificant $(P=0.2423)$. The highest PDI value observed was $(0.323 \mathrm{Ð})$ for particles with drug/polymer ratio $1: 1$ and was significantly different from the other two values $(P=0.0005)$.

3.3. Zeta Potential. Zeta potential of the produced nanoparticles was significantly different among all the three samples at 5\% standard error $(P=0.001, P=0.01$, and $P=0.0001$ for the comparison of particles with drug/polymer ratios $1: 1$ and $1: 2,1: 1$ and $1: 4$, and $1: 2$ and $1: 4$, resp.). The highest zeta potential $(5.98 \mathrm{mV})$ was observed for the particles with drug/polymer ratio $1: 4$ and the lowest value $(3.31 \mathrm{mV})$ was observed for the particles with drug/polymer ratio $1: 2$.

3.4. In Vitro Drug Release. The drug release profile was biphasic for the sample with drug/polymer ratio $1: 1$. This then shifted towards monophasic as the drug/polymer ratio decreased to $1: 4$ (Figure 1).

\section{Discussion}

Drug encapsulation into nanoparticles modifies the drug's pharmacokinetics by masking its physicochemical properties. In turn, the nanoparticle characteristics determine the pharmacokinetics and stability of the drug. In vitro characterization of nanoparticles gives a prediction of a drug's pharmacokinetics in physiological fluids so as to improve its stability in a formulation.

High encapsulation efficiency observed for particles with drug/polymer ratio 1: 4 (Table 1) is favorable. High encapsulation efficiency minimizes drug wastage during the fabrication process. It also ensures high drug concentration at the target site and increased drug residence time.

Other studies have however recorded that encapsulation efficiency of water soluble drugs can be as high as $80 \%$ to $100 \%$ when the double emulsion evaporation method is used [8].

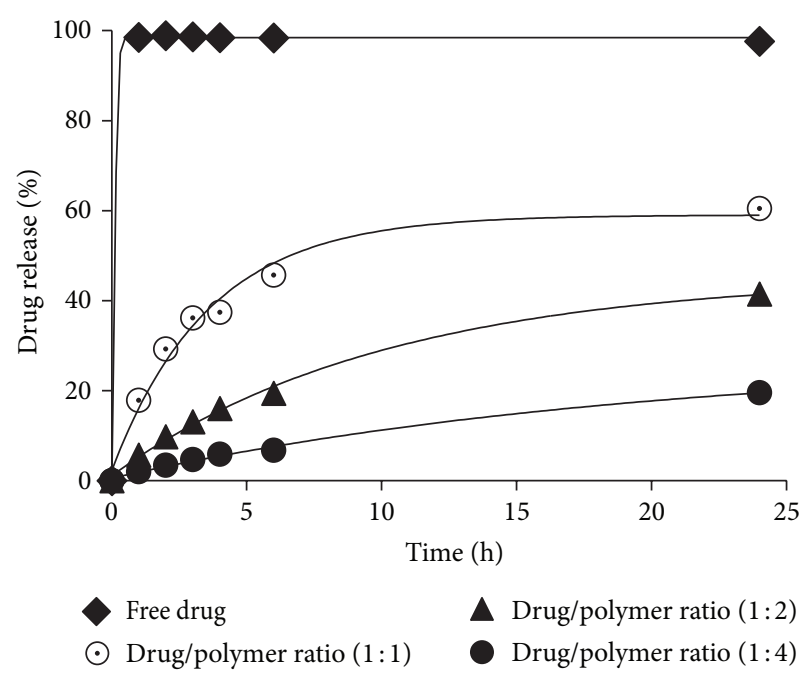

FIGURE 1: Graph of drug release profile of pralidoxime-loaded PLGA nanoparticles in comparison with the free drug.

The reduced encapsulation efficiency observed in this study (69.27\%) can be attributed to low PLGA concentration in the dispersed phase [9]. This leads to a less viscous solution which leads to slow precipitation of the PLGA at the dispersed phase surface, resulting in increased drug diffusion across the phase boundary and low encapsulation consequently [9]. High pralidoxime solubility in water (the continuous phase) is also a factor that reduces encapsulation efficiency [10]. Other possible contributing factors include high PLGA solubility in ethyl acetate, low ratio of the dispersed phase to continuous phase (encapsulation efficiency increases with increase in the volume of the continuous phase), high stirring rate during fabrication in an effort to minimize the final particle size, and high PVA concentration which was used as an emulsifier $[10,11]$.

The decrease in encapsulation efficiency with increase in drug/polymer ratio was in line with the results of studies by Trivedi and colleagues [12] as well as Nagda and colleagues [11]. In high drug/polymer ratios, the polymer layer surrounding the drug molecules is thin; hence the drug can easily escape encapsulation before polymer solidification unlike in the presence of low drug/polymer ratio where the diffusion layer is thick.

Particle size is an important factor to consider in circumvention of the blood brain barrier. Small particles are of great interest as opposed to larger particles that would be trapped in the tight junctions [13]. Moreover, nanoparticles for central nervous system drug delivery should not have a higher tendency of aggregation. The degree of nanoparticle aggregation in a liquid medium can be determined by the polydispersity index, values close to zero being favorable as opposed to those close to one, which indicate a high degree of aggregation.

The particles produced for all the three samples with different drug/polymer ratios were small (in the nanometer range) and formed monodispersion in water; PDI $<0.5$ (Table 2) means that there was a uniform size and shape 
distribution for the particles in each sample. Monodispersion favors cellular uptake of the nanoparticles as opposed to aggregated particles (the presence of which can be shown by a high PDI) which interferes with it due to the big size.

Though cell membranes allow a free passage of particles less than $1 \mathrm{~nm}$ in diameter [14], because of their size, the nanoparticles can potentially enhance pralidoxime delivery into the central nervous system by increasing pralidoxime concentration inside or at the BBB cell luminal surface. Thus they create a higher concentration gradient between the blood and brain than the one obtainable after systemic administration of the free drug [15]. The drug's pharmacokinetics can also be altered to favor the central nervous system delivery by increased circulation time of the drug in the blood stream as systems such as mononuclear phagocyte system only tag and remove particles with a minimal diameter of 1 micrometer from the circulation [16]. In vivo studies might however be necessary to ascertain this.

Much smaller pralidoxime-loaded PLGA nanoparticles could be obtained. One of the reasons that could have resulted in the recorded particles size might be the shearing method used (homogenization) which was recorded to be associated with large particles [17]. The use of ultrasonication (using a probe sonicator) could have possibly resulted in even much smaller particles.

For the three samples with different drug/polymer ratios, zeta potential values were small (less than 20) (Table 2). The low values can be an indication of how the pralidoxime is encapsulated in PLGA nanoparticles, shielded in the polymer not adsorbed at the polymer surface [18]. Low zeta potential values are however associated with low stability of particles due to low electrostatic repulsive forces between the particles; consequently, the particles will have a high tendency of aggregating which in turn compromises the stability of the particles in a formulation [19].

On the contrary, small PDI values (close to zero) (Table 2) show that all the samples formed monodispersions in water, a scenario which is impossible in the presence of aggregating. This suggests that the particles were stable despite the low zeta potential values. Since PLGA is a large molecule (polymer), the stability could be more due to steric hindrance than surface charge [19]. Moreover, an adsorbed layer of a polymer or large molecular weight molecule tends to shift plane of shear further from the particle surface and, as a result, the measured zeta potential decreases [20]. This means that, even in the case of highly charged particles, a relatively low zeta potential value can be recorded.

The nature of the recorded zeta potential values (small and positively charged) can improve the pharmacokinetics of the drug by increasing the circulation time of the nanoparticles in the blood [21]. Negatively charged nanoparticles are quickly cleared from the blood stream by fixed macrophages of the reticuloendothelial system [22]. Likewise, highly surface charged particles are efficiently phagocytized by murine macrophages more than those with small charge [21].

For the drug to be released from the PLGA nanoparticles, the PLGA undergoes degradation by hydrolysis or biodegradation through cleavage of its backbone ester linkage into oligomers and finally monomers [23]. The clear biphasic release profile of the sample with drug/polymer ratio $1: 1$ indicates the presence of pralidoxime close to or at the PLGA surface [24]. This accounts for the initial burst in drug release as the drug gets in contact with water. The rapid release is related to pralidoxime solubility and water penetration into the PLGA matrix [25]. Subsequently, the graph shows a delayed release profile. This can be attributed to the progressive pralidoxime release through the thicker pralidoxime depleted layer [25]. Water inside the drug matrix hydrolyses the PLGA into its oligomeric then monomeric soluble products [23]. The drug then freely passes and is released by erosion and diffusion until there is a complete PLGA solubilization. The biphasic release profile might be favourable since it can be used for controlled drug release.

The first phase (rapid release) is less pronounced for the sample with drug/polymer ratio $1: 2$. The delayed release phase is dominant. This indicates that there is little pralidoxime close to the PLGA surface [23]. For the sample with drug/polymer ratio $1: 4$, the initial rapid release phase is invisible on the graph (Figure 1). This shows that pralidoxime is completely shielded by PLGA and no drug molecules are close to the polymer surface. This type of drug release (monophasic but sustained) may be convenient to the patient as it reduces dosing frequency and increases compliance. However, it takes time before the onset of action is experienced, something undesirable in managing a poisoned patient. Therefore, a loading dose might be necessary in this case or initiating this drug in combination with another organophosphate antidote, for example, atropine.

\section{Conclusion}

The pralidoxime-loaded PLGA nanoparticles were produced by the double emulsion solvent evaporation method with the highest encapsulation efficiency being 68.78\% (for particles with drug/polymer ratio 1:4), lowest mean particle size of $304.7 \mathrm{~nm}$ (from particles with drug/polymer ratio 1:2 though not statistically different from the size recorded by the particles with drug/polymer ratio 1:4), and the highest zeta potential being $5.98 \mathrm{mV}$ (recorded for particles with drug/polymer ratio 1:4). The in vitro drug release changed from biphasic to monophasic from the drug/polymer ratios $1: 1$ to $1: 4$. Generally, the drug/polymer ratio $1: 4$ produced better results compared to the ratios $1: 1$ and $1: 2$.

\section{Conflict of Interests}

The authors declare that there is no conflict of interests regarding the publication of this paper.

\section{Acknowledgments}

The authors would like to thank technicians at the Western Cape University (South Africa) for assistance in the in particle size, PDI, and zeta potential characterization. The authors also acknowledge the following departments at the University of Zimbabwe for their laboratories and equipment: Department of Biochemistry, Department of Physiology, and Faculty of Veterinary Sciences. We also acknowledge the Innovation 
and Commercialization (ICF) grant received for the purpose of developing this study.

\section{References}

[1] Y. Li, C. Sun, Z. Qiu et al., "Clinical toxicology in China: current situation and future development," Clinical Toxicology, vol. 47, no. 4, pp. 263-269, 2009.

[2] M. Eddleston, N. A. Buckley, P. Eyer, and A. H. Dawson, "Management of acute organophosphorus pesticide poisoning," The Lancet, vol. 371, no. 9612, pp. 597-607, 2008.

[3] N. J. Secaucus and Occupational Health Services, MSDS for Methyl Parathion, OHS, 1991.

[4] M. Balali-Mood and K. Balali-Mood, "Neurotoxic disorders of organophosphorus compounds and their managements," Archives of Iranian Medicine, vol. 11, no. 1, pp. 65-89, 2008.

[5] G. M. Tush and M. I. Anstead, "Pralidoxime continuous infusion in the treatment of organophosphate poisoning," Annals of Pharmacotherapy, vol. 31, no. 4, pp. 441-444, 1997.

[6] R. I. Ellyn and J. H. Wills, "Oximes antagonistic to inhibitors of cholinesterase," Journal of pharmaceutical sciences, vol. 53, pp. 995-1007, 1964.

[7] Y. E. Choonara, V. Pillay, V. M. K. Ndesendo et al., "Polymeric emulsion and crosslink-mediated synthesis of superstable nanoparticles as sustained-release anti-tuberculosis drug carriers," Colloids and Surfaces B: Biointerfaces, vol. 87, no. 2, pp. 243-254, 2011.

[8] M. Iwata and J. W. McGinity, "Preparation of multi-phase microspheres of poly(D,L-lactic acid) and poly(D,L-lactic-coglycolic acid) containing a W/O emulsion by a multiple emulsion solvent evaporation technique," Journal of Microencapsulation, vol. 9, no. 2, pp. 201-214, 1991.

[9] H. Rafati, A. G. A. Coombes, J. Adler, J. Holland, and S. S. Davis, "Protein-loaded poly(DL-lactide-co-glycolide) microparticles for oral administration: formulation, structural and release characteristics," Journal of Controlled Release, vol. 43, no. 1, pp. 89-102, 1997.

[10] R. C. Mehta, B. C. Thanoo, and P. P. DeLuca, "Peptide containing microspheres from low molecular weight and hydrophilic poly(d,l-lactide-co-glycolide)," Journal of Controlled Release, vol. 41, no. 3, pp. 249-257, 1996.

[11] C. D. Nagda, N. P. Chotai, S. B. Patel, T. J. Soni, and U. L. Patel, "Preparation and in vitro evaluation of bioadhesive microparticulate system," International Journal of Pharmaceutical Sciences and Nanotechnology, vol. 1, no. 3, pp. 257-266, 2008.

[12] P. Trivedi, A. Verma, and N. Garud, "Preparation and characterization of aceclofenac microspheres," Asian Journal of Pharmaceutics, vol. 2, no. 2, pp. 110-115, 2008.

[13] J. Panyam, W.-Z. Zhou, S. Prabha, S. K. Sahoo, and V. Labhasetwar, "Rapid endo-lysosomal escape of poly(DL-lactideco-glycolide) nanoparticles: implications for drug and gene delivery," The FASEB Journal, vol. 16, no. 10, pp. 1217-1226, 2002.

[14] P. R. Lockman, R. J. Mumper, M. A. Khan, and D. D. Allen, "Nanoparticle technology for drug delivery across the blood brain barrier," Drug Development and Industrial Pharmacy, vol. 28, no. 1, pp. 1-13, 2002.

[15] M. Masserini, "Nanoparticles for brain drug delivery," ISRN Biochemistry, vol. 2013, Article ID 238428, 18 pages, 2013.

[16] R. L. McCall and R. W. Sirianni, "PLGA nanoparticles formed by single- or double-emulsion with vitamin E-TPGS," Journal of Visualised Experiments, vol. 82, Article ID e51015, pp. 1-8, 2013.
[17] B. N. Fredricksen, PLGA and PLA particles as vaccine delivery systems for Atlantic salmon [Ph.D. thesis], University of Tromso, 2012.

[18] N. Csaba, M. Garcia-Fuentes, and M. J. Alonso, "The performance of nanocarriers for transmucosal drug delivery," Expert Opinion on Drug Delivery, vol. 3, no. 4, pp. 463-478, 2006.

[19] S. Honary and F. Zahir, "Effect of zeta potential on the properties of nano-drug delivery systems-a review (Part 2)," Tropical Journal of Pharmaceutical Research, vol. 12, no. 2, pp. 265-273, 2013.

[20] F. Quaglia, L. Ostacolo, A. Mazzaglia, V. Villari, D. Zaccaria, and M. T. Sciortino, "The intracellular effects of non-ionic amphiphilic cyclodextrin nanoparticles in the delivery of anticancer drugs," Biomaterials, vol. 30, no. 3, pp. 374-382, 2009.

[21] C. He, Y. Hu, L. Yin, C. Tang, and C. Yin, "Effects of particle size and surface charge on cellular uptake and biodistribution of polymeric nanoparticles," Biomaterials, vol. 31, no. 13, pp. 36573666,2010

[22] M. K. Khan, S. S. Nigavekar, L. D. Minc et al., "In vivo biodistribution of dendrimers and dendrimer nanocompositesimplications for cancer imaging and therapy," Technology in Cancer Research and Treatment, vol. 4, no. 6, pp. 603-613, 2005.

[23] H. K. Makadia and S. J. Siegel, "Poly Lactic-co-Glycolic Acid (PLGA) as biodegradable controlled drug delivery carrier," Polymers, vol. 3, no. 3, pp. 1377-1397, 2011.

[24] L. C. Amann, M. J. Gandal, R. Lin, Y. Liang, and S. J. Siegel, "In vitro-in vivo correlations of scalable PLGA-risperidone implants for the treatment of schizophrenia," Pharmaceutical Research, vol. 27, no. 8, pp. 1730-1737, 2010.

[25] M. Ramchandani and D. Robinson, "In vitro and in vivo release of ciprofloxacin from PLGA 50:50 implants," Journal of Controlled Release, vol. 54, no. 2, pp. 167-175, 1998. 

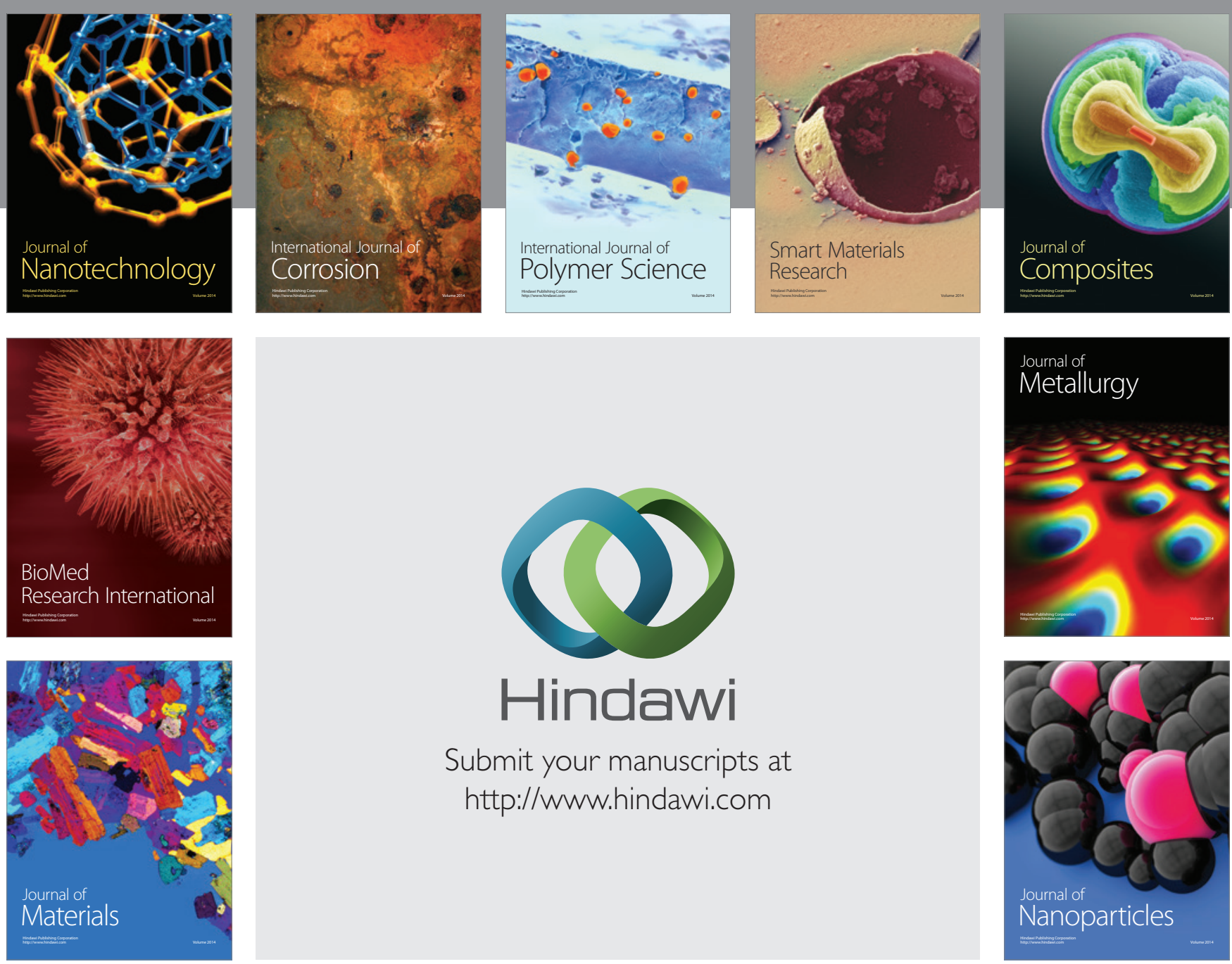

Submit your manuscripts at http://www.hindawi.com
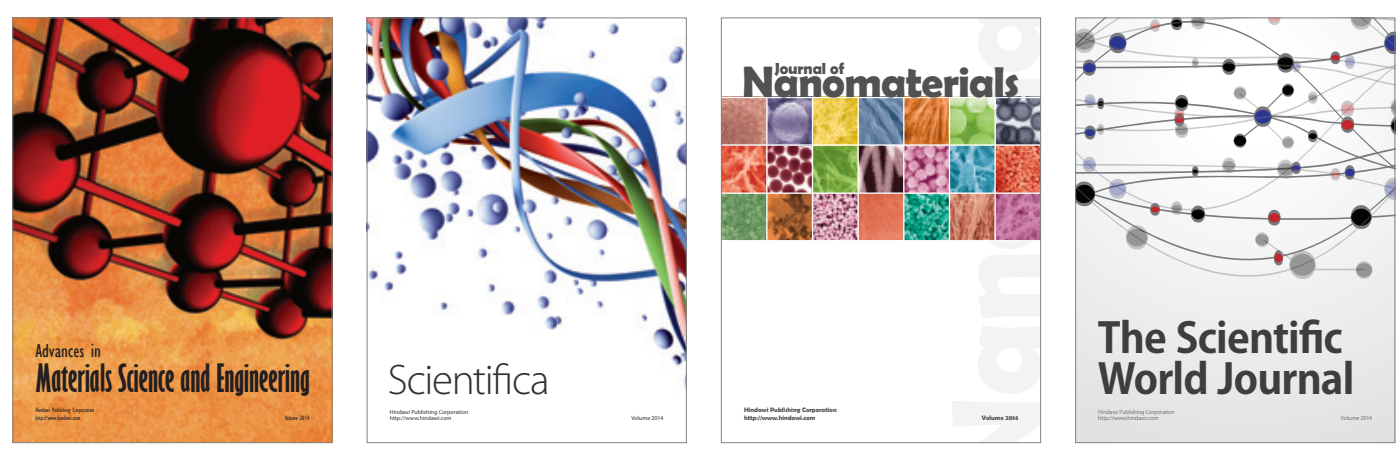

\section{The Scientific World Journal}
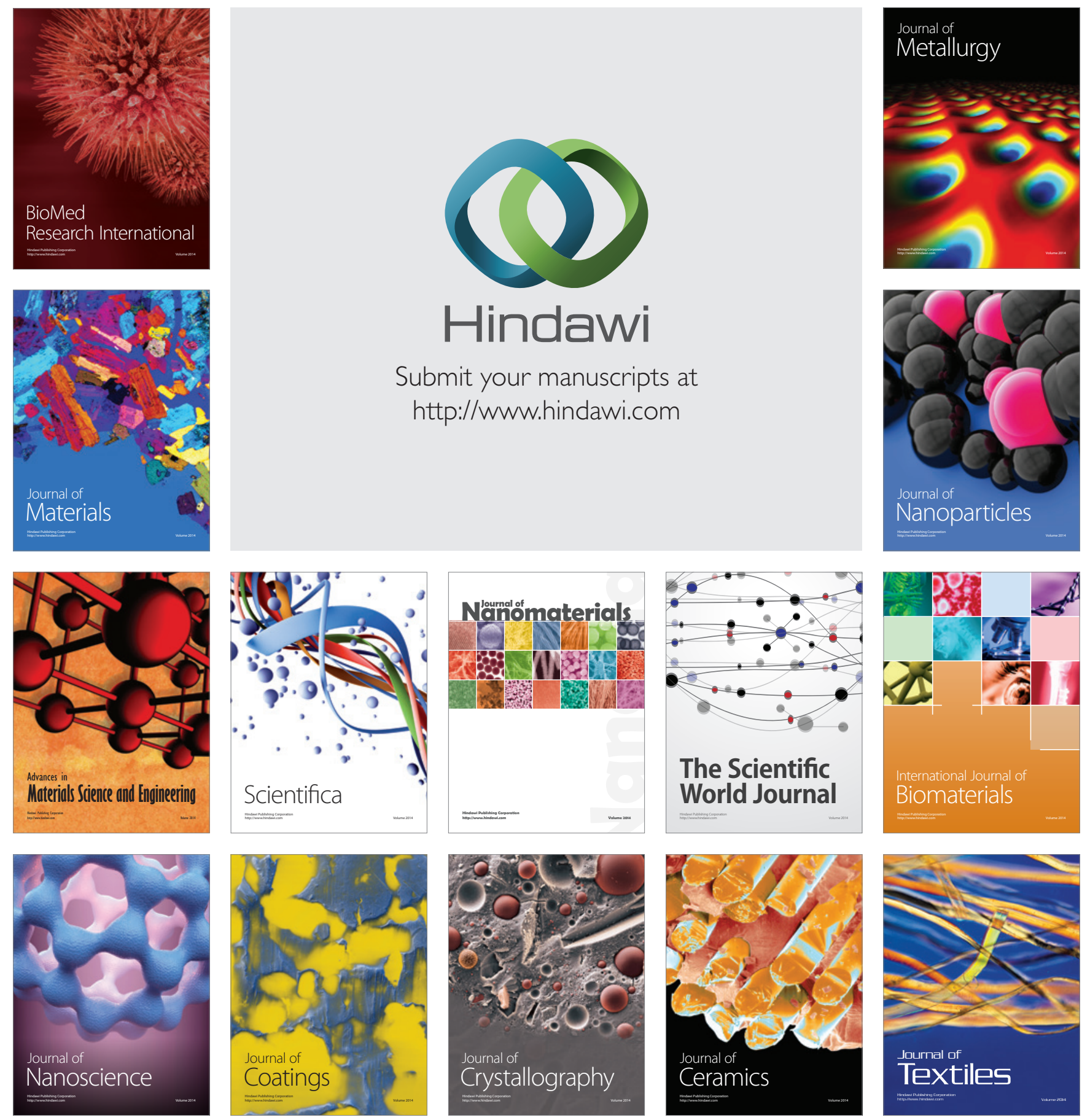